\title{
Gamma-Ray Observations with CALET: Exposure Map, Response Functions, and Simulated Results
}

\author{
Nicholas Cannady ${ }^{* \dagger}$ and \\ Department of Physics and Astronomy, Louisiana State University, Baton Rouge, LA, 70803, \\ USA \\ E-mail: ncannalelsu.edu \\ Michael L. Cherry for the CALET Collaboration \\ Department of Physics and Astronomy, Louisiana State University, Baton Rouge, LA, 70803, \\ USA \\ E-mail: cherry@phys.1su.edu
}

The CALorimetric Electron Telescope (CALET) is a space-borne cosmic ray instrument planned for installation on the JEM-EF platform on the International Space Station (ISS) in 2015. The CALET collaboration is a Japan-led international team involving researchers in Italy and the U.S. In addition to precise measurement of the cosmic ray electron and nuclei spectra, the CALET calorimeter will be capable of gamma-ray observations in the $\mathrm{GeV}$ to tens of $\mathrm{TeV}$ energy range. This paper presents a study of the expected gamma-ray signal measured by CALET in the first year on orbit. The ISS zenith pointing is simulated at a time resolution of 1 second in order to estimate the exposure map on the sky. The instrument response functions and simulated results of gamma-ray/electron separation for the calorimeter are discussed and used to estimate the expected point source and galactic diffuse signals in the energy range $\sim 10 \mathrm{GeV}-\sim 500 \mathrm{GeV}$ based on known fluxes measured by Fermi-LAT.

The 34th International Cosmic Ray Conference,

30 July- 6 August, 2015

The Hague, The Netherlands

\footnotetext{
* Speaker.

${ }^{\dagger}$ CALET supported in the USA by NASA grant \#NNX11AE01G
} 


\section{Introduction}

The CALorimetric Electron Telescope (CALET), a Japanese-led cosmic-ray experiment with Italian and US collaborators, is scheduled for deployment on the International Space Station (ISS) in the middle of 2015 for high-accuracy measurement of electrons above $10 \mathrm{GeV}$ and up to and beyond $10 \mathrm{TeV}$, as well as protons, heavier nuclei, and gamma-rays [1].

This paper examines the expected gamma-ray signal in CALET after 1 year of on-orbit data collection. An algorithm is developed and evaluated for the separation of a gamma-ray event dataset from the electron dataset based on simulations performed with the EPICS and Cosmos Monte Carlo code [2]. An exposure map is generated for the 1-year CALET orbit on the ISS and presented along with the resulting expectations of gamma-ray counts. Source fluxes and background modelling are based upon recent Fermi-LAT results.

\section{The CALET calorimeter}

The CALET CALorimeter (CAL) is the primary detector on the CALET mission. It is composed of three main components: the CHarge Detector (CHD), the IMaging Calorimeter (IMC), and the Total AbSorption Calorimeter (TASC). The CHD is composed of two crossed layers of plastic scintillator strips with the purpose of measuring the ionization energy deposit of primary particles entering the CAL. The IMC is eight layers, each made up of a pair of crossed layers of $1 \mathrm{~mm}^{2}$ plastic scintillator fibers. Interspersed between the eight layers are thin sheets of tungsten. With a total depth of $3 \mathrm{X}_{0}$ for normal incidence, the IMC will be capable of detailed measurement of the early shower development for high-precision reconstruction of the primary particle trajectory. The TASC contains 12 layers of PWO logs alternating in xy-orientation (total thickness $27 \mathrm{X}_{0}$ for normal incidence), which will absorb the majority of the energy deposited by particle showers [1]. A schematic of the CAL is shown in Figure 1 without any of the shielding that is present in the Monte Carlo simulations.

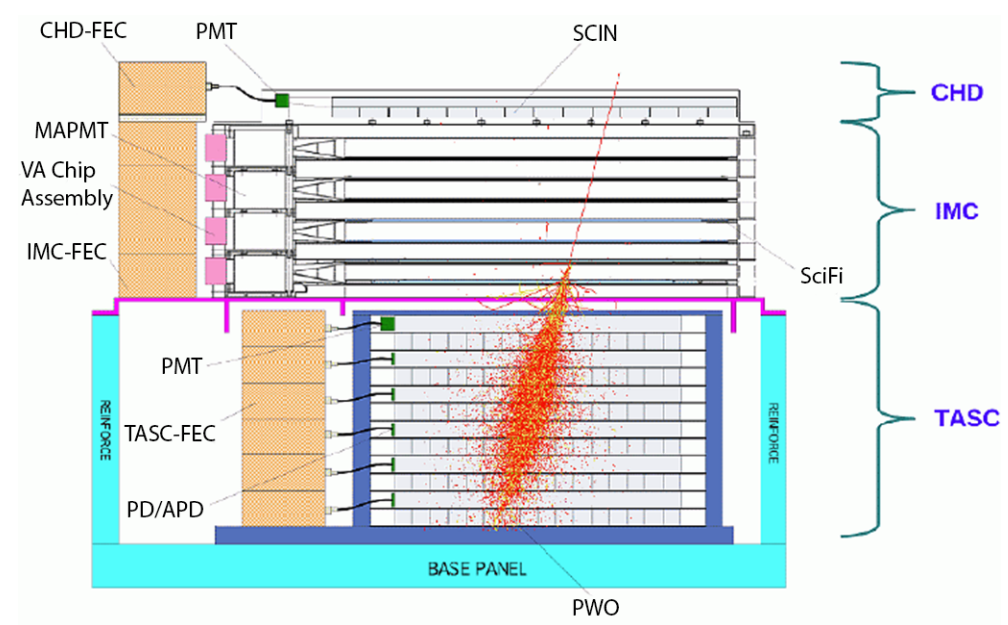

Figure 1: Simulation of a $1 \mathrm{TeV}$ electron in the CAL. 


\section{Gamma-ray/electron separation}

Distinguishing between primary electrons and photons will be necessary for any CALET gamma-ray science goals. The algorithm developed and described in this section is based only on the signal in those CHD strips which were directly passed through by the primary particle, henceforth referred to as "hit" components. The trajectories themselves are not reconstructed, but taken directly from the Monte Carlo truth. The data used for the following results are generated by the EPICS and Cosmos Monte Carlo simulation package on the LSU High Performance Computing SuperMike-II cluster.

Gamma-ray and electron events were generated isotropically on a partial sphere $\left(\theta \leq 110^{\circ}\right)$ with an $\mathrm{E}^{-1}$ spectrum in three energy bins: $10-100 \mathrm{GeV}, 100-1000 \mathrm{GeV}, 1-10 \mathrm{TeV}$. Events were generated for each species in each of these energy bins and subsequently filtered based on their trajectories. The requirements placed on events for analysis follow the "type B" geometry as defined in [3]. Explicitly, this enforces that the primary particle passes through both the top of the CHD and the bottom of the TASC.

For each of the type B events, the energy deposits in the hit strips in CHDx and CHDy were summed, respectively. Histograms of these deposits (see Figure 2) verify that the distribution for electrons appears as a minimum ionizing particle (MIP) distribution, whereas the gamma-rays primarily exhibit zero energy deposit. Some gamma-rays pair produce in the aluminum structure above the detector or in the CHD itself, leading to partial energy deposits due to secondary electrons and a feature around $4 \mathrm{MeV}$ (2 MIP) clearly seen in the $10-100 \mathrm{GeV}$ and $100-1000 \mathrm{GeV}$ histograms. The pair production cross section and backscatter pollution of the CHD signal increase with photon energy, leading to an increasing fraction of non-zero energy deposits in the CHD at higher energies.

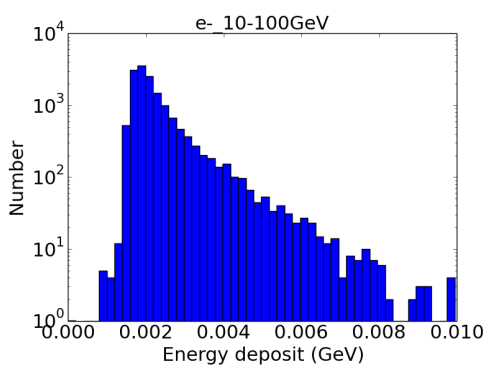

10-100 GeV electrons

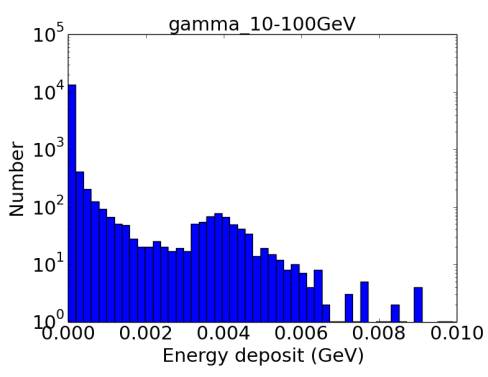

10-100 GeV gamma-rays

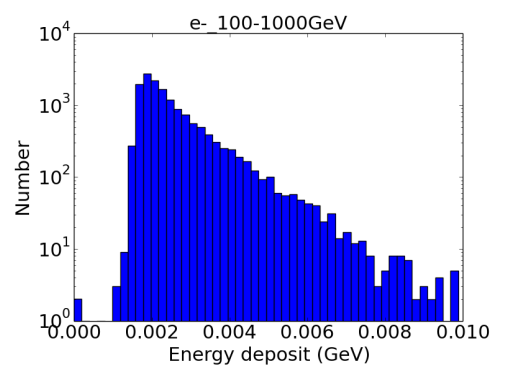

100-1000 GeV electrons

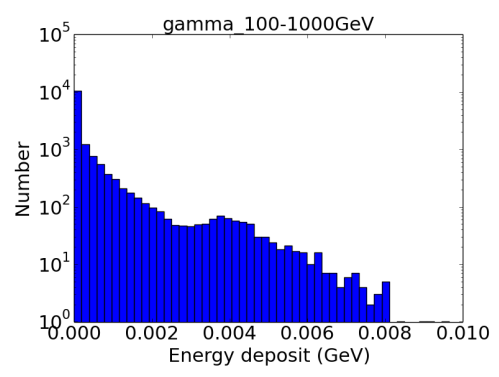

100-1000 GeV gamma-rays

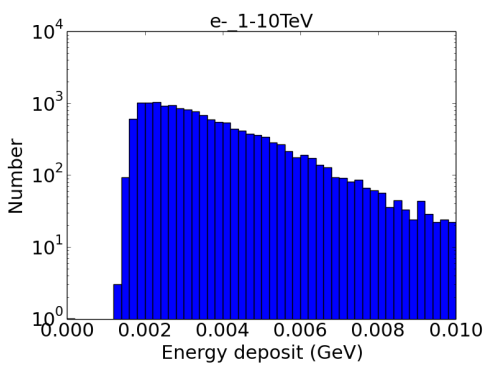

1-10 TeV electrons

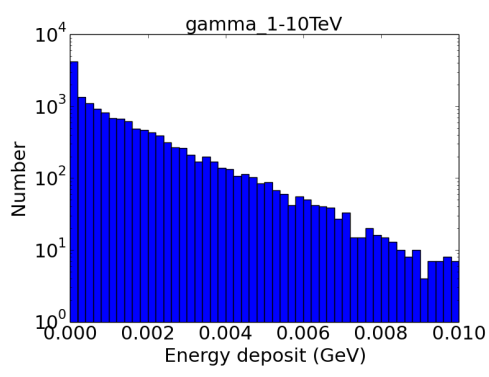

1-10 TeV gamma-rays

Figure 2: Histograms of CHD energy deposits in each energy bin, summed over hit strips in the layer. 

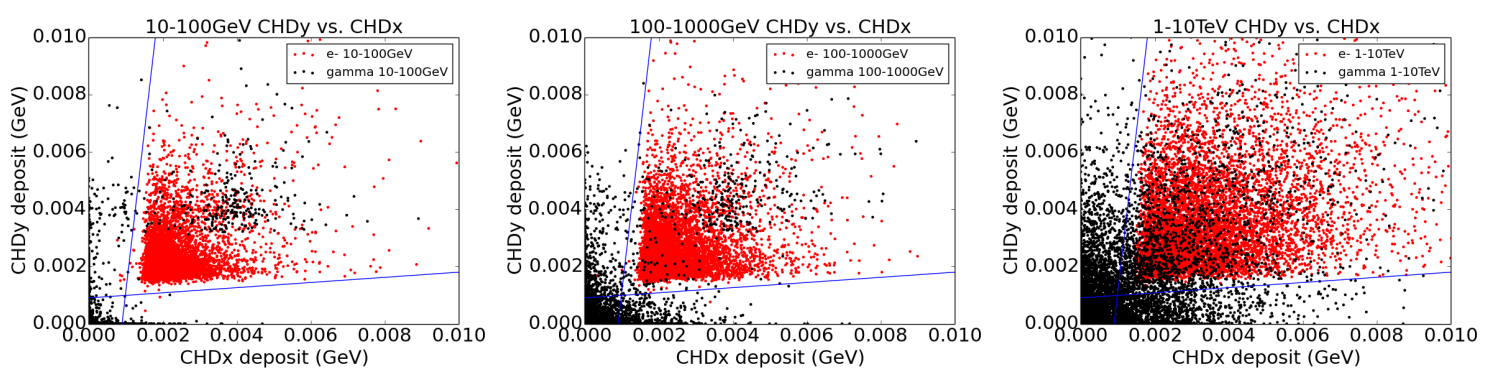

Figure 3: Energy deposits in CHDy vs. CHDx for both electrons and gammas in each energy range. The blue lines indicate the cuts made to separate the samples.

In the $1-10 \mathrm{TeV}$ histogram, the $4 \mathrm{MeV}$ feature is indistinguishable from the continuum due to the large fraction of interacting gamma-rays and energy deposits from backscattered particles.

Scatter plots showing the total energy deposited in the hit components of CHDy as a function of the total energy deposited in the hit components of CHDx provide a graphical representation of the cuts used to separate the gamma-rays from the electrons. As can be seen in Figure 3, two straight lines (one nearly horizontal, the other nearly vertical) can be drawn which largely divide the samples. The functional requirement for identifying a gamma-ray by this scheme can be written

$$
\mathrm{CHDx} \_\mathrm{dE} \leq 0.09 \times\left(\mathrm{CHDy} \_\mathrm{dE}+0.01\right) \quad \text { or } \quad \mathrm{CHDx} \_\mathrm{dE} \geq\left(\mathrm{CHDy} \_\mathrm{dE} / 0.09\right)-0.01
$$

Table 1: Efficiency for separation of electrons and gamma-rays in type B geometry into individual datasets

\begin{tabular}{|c|c|c||c|c|c|c|}
\hline Species & Energy & \# in-geom. & \# ID as $\gamma$ & $\%$ ID as $\gamma$ & \# ID as e & $\%$ ID as e \\
\hline \multirow{4}{*}{ e- } & $10-100 \mathrm{GeV}$ & 7675 & 8 & $0.104 \%$ & 7667 & $99.9 \%$ \\
& $100-1000 \mathrm{GeV}$ & 7554 & 5 & $0.0662 \%$ & 7549 & $99.9 \%$ \\
& $1-10 \mathrm{TeV}$ & 7485 & 5 & $0.0668 \%$ & 7480 & $99.9 \%$ \\
\hline$\gamma$ & $10-100 \mathrm{GeV}$ & 7482 & 7217 & $96.5 \%$ & 265 & $3.54 \%$ \\
& $100-1000 \mathrm{GeV}$ & 7649 & 7275 & $95.1 \%$ & 374 & $4.89 \%$ \\
& $1-10 \mathrm{TeV}$ & 7573 & 5797 & $76.5 \%$ & 1776 & $23.5 \%$ \\
\hline
\end{tabular}

The criterion in Eq. 3.1 was applied to all of the type B events in order to characterize the loss of gamma-rays from the sample and the rejection of electrons. The statistical results are summarised in Table 1.

It should be emphasized that these results are as of yet preliminary, and only encompass events of type A or B. Further work is in progress to extend this study to type $\mathrm{C}$ and $\mathrm{D}$ events using deposits in the IMC as well. Using the IMC signals, tracking of the primary particle can be determined instead of using the Monte Carlo truth. This trajectory and the associated error can be used to identify those IMC fibers which could have been passed through by the primary particle. The energy deposits in these fibers will show an ionization deposit if the primary particle is an electron, which can be used to further improve the electron rejection power. These calculations will also provide good estimates of the effect of trajectory reconstruction error on the ability to make a clean separation. 


\section{CALET 1-year exposure map}

When deployed on the ISS, CALET will always be oriented toward the zenith [3]. It has a $>45^{\circ}$ field of view radius and a high orbital inclination, and will, as such, be an all-sky monitor of high-energy gamma-rays. In order to estimate the number of photons from known sources (diffuse galactic emission, isotropic emission, and persistent point sources), a map of exposure time on the sky has been generated. The resolution of the sky map was chosen to match the Fermi-LAT galactic diffuse background model [4], which is binned to $0.125^{\circ} \times 0.125^{\circ}$ in galactic coordinates. Orbital ephemeris information was generated for the ISS orbit for a full year at $1 \mathrm{~s}$ resolution using the AGI Satellite Toolkit (STK) software. The calculated pointing directions were transformed to galactic coordinates and used to determine the total duration for which each bin on the sky would be within the field of view of the instrument. The resulting exposure map is shown in Figure 4.

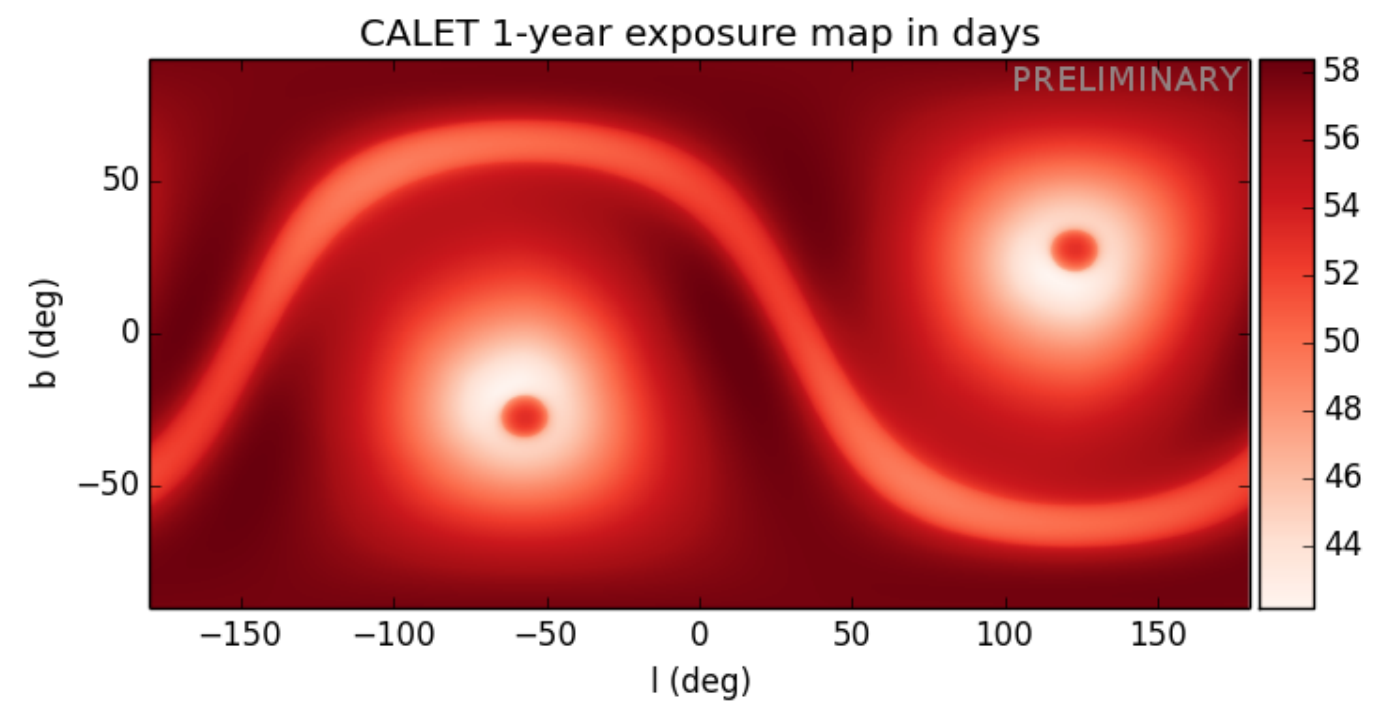

Figure 4: CALET exposure map for continuous observation from Nov. 1, 2015 through Oct. 31, 2016. Sharp features are due to the not yet accounted for zenith angle dependence of the instrument response.

\section{Estimated number of events}

In order to calculate the expected number of photons incident on the calorimeter, the FermiLAT galactic diffuse and isotropic background models are used, as well as the Fermi-LAT 1FHL and 2FGL catalogs for the persistent point sources. The instrument response functions used in the following calculations are taken from [3]. The effective area $\mathrm{A}_{\text {eff }}$ of the CAL is a function of energy and zenith angle of the incident radiation. Since angular information was not simulated for the following calculations, the effective area is averaged over the field of view. The energy dependence is preserved, and is taken into account by the integration of $\mathrm{A}_{e f f}$ against the fluxes. The energy dependence of $\mathrm{A}_{\text {eff }}$ reflects $100 \%$ detection above $10 \mathrm{GeV}$, decreasing quickly to $50 \%$ at $5 \mathrm{GeV}$ and zero detection below $2.5 \mathrm{GeV}$. The exposure time $\mathrm{t}_{\text {exp }}$ is taken for each bin from the generated exposure map in Figure 4. The solid angle of the each sky bin can be calculated as a function of the bin center galactic latitude. 


\subsection{Diffuse galactic and isotropic}

The Fermi-LAT background models [4] give fluxes for the isotropic and galactic diffuse gamma-ray signals. Since the energies are binned differently for the two models and the background spectra are not within the scope of this paper, the contributions from all energy bins above $2.5 \mathrm{GeV}$, including the effects of decreasing efficiency at lower energies (reaching up to 500 $\mathrm{GeV}$ for galactic sources and up to $600 \mathrm{GeV}$ for the isotropic signal) are summed to obtain a total number of background photons in each sky bin. Summing the contributions from every sky bin, we calculate that CALET will detect approximately 7500 in-geometry photons at energies $\geq 2.5 \mathrm{GeV}$ from the known diffuse flux per year, with about 2500 of those at energies $>10 \mathrm{GeV}$.

\subsection{Persistent sources}

The estimate of counts above $2.5 \mathrm{GeV}$ from point sources was taken from the first FermiLAT catalog of high-energy gamma-ray sources [5] and the second Fermi-LAT source catalog [6]. Extended sources which subtend less than $2^{\circ}$ are included in this catalog, while larger extended sources are contained in the diffuse galactic background model [4]. All sources with more than three incident photons at energies $>10 \mathrm{GeV}$ are also shown on a sky map in Figure 5. The total number of photons expected from discrete photons is on the order of 1000, including contributions from Vela (190 photons), Geminga (110 photons), and the Crab (44 photons).

The photons from these sources will be spread across several sky bins, as a conservative estimate of the PSF has a $1^{\circ}$ diameter. The background in each individual sky bin was calculated, considering both the diffuse gamma-ray emission and the contamination from electrons (flux taken as upper limit of PAMELA results for the $10-600 \mathrm{GeV}$ range [8], then reduced by the appropriate electron rejection factor), and found to be negligible compared to the signal from these sources.

The estimated number of gamma-rays above reflect the expected CALET energy threshold for $100 \%$ detection of $10 \mathrm{GeV}$. Similar estimates have been previously for a reduced threshold of 1 GeV [7] for Vela ( 2100), Geminga ( 1200), and the Crab ( 360). The discrepancy between the numbers presented in this work and those in [7] result from differences in the effective area and efficiency used for each study. It is calculated in [7] that lowering the energy threshold from 10 $\mathrm{GeV}$ to $5 \mathrm{GeV}$ would result in a factor of 5-10 increase in the number of detected gamma-rays from persistent sources.

\section{Observation of transient systems}

A significant strength of CALET for high-energy gamma-rays is the large orbital inclination of the ISS and the large field of view. These properties make CALET very well-suited as an all-sky monitor for high-energy flaring activity. It is possible, as mentioned in [7], that the energy threshold will be lowered to $1-5 \mathrm{GeV}$ rather than the $10 \mathrm{GeV}$ used in this paper. Not only would a decrease in threshold to $5 \mathrm{GeV}$ increase the number of detected photons from persistent point sources by a factor of 5-10, it would also improve CALET's performance as a monitor for transient systems.

As an example, the expected number of photons was calculated for two historical high-energy Crab flares. The flares will be characterized by their duration $(\tau)$ and the ratio of the average flux during the flare to the ambient flux $(\alpha)$. For the present work, the flare spectrum has not 


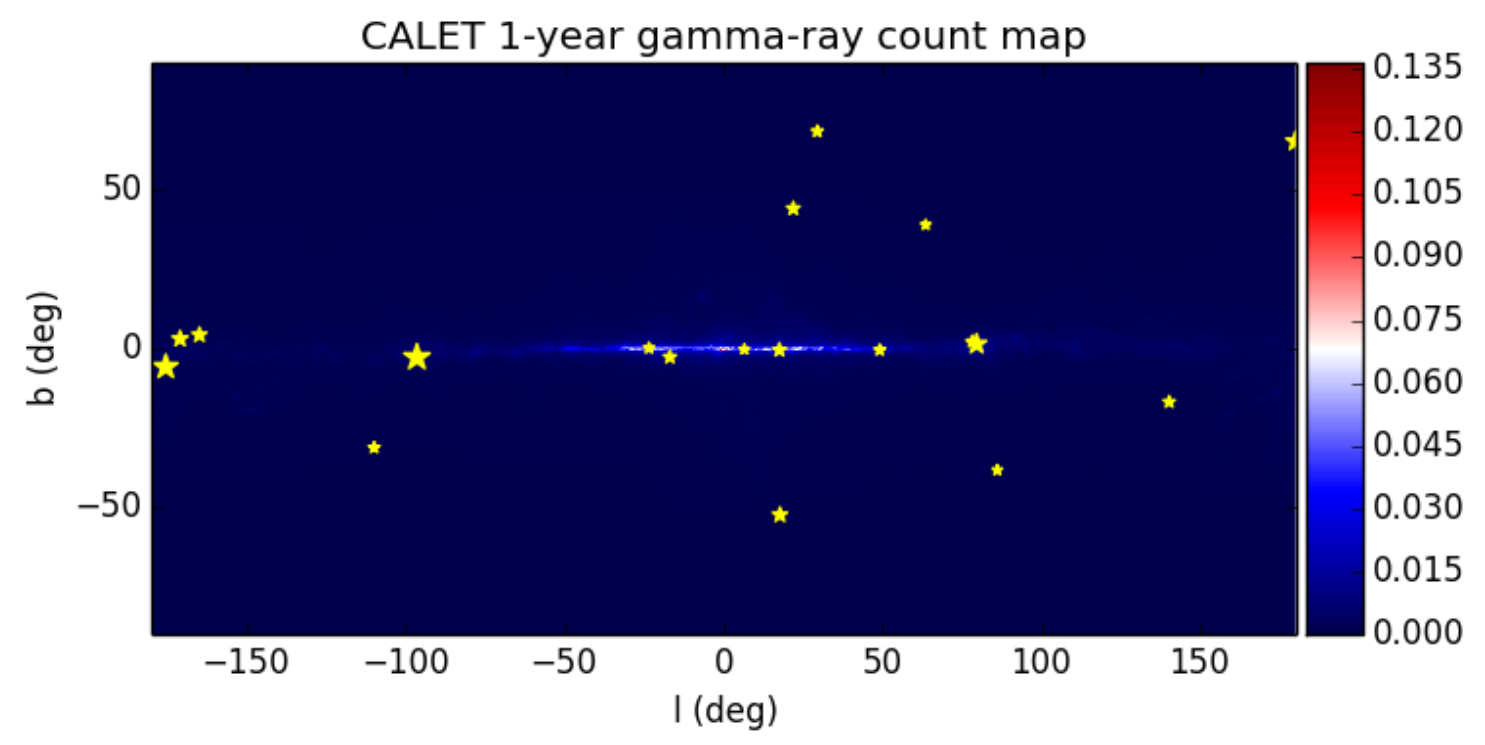

Figure 5: 1-year count map for CALET gamma-rays. The background color represents intensity of diffuse emission in the area. The stars are steady point sources, with the size of the marker proportional to the number of photons. Only sources with $\geq 3$ photons for $\mathrm{E}>10 \mathrm{GeV}$ are shown on the plot.

been accounted for, and the following numbers reflect a uniform enhancement of the normal Crab spectrum. For the April 2011 flare, $\tau \approx 10$ days and $\alpha \approx 30$. [9], and for the March 2013 flare, $\tau \approx$ 20 [10]. If a flare were to occur on a day where CALET could ideally observe it, the exposure time is approximately 12600 seconds ( $25 \%$ of the full day). The expected signal from an $\alpha=25$ flare is then $N \approx 3$ photons. For the historical flares, the fractional exposure time is taken to be $16 \%$ of the total flare time, which is the ratio of the exposure time calculated for the Crab in the orbit simulation to the total time in one year. These parameters yield an estimate of 35 photons over the full flaring period for either flare. This translates to a $\sim 6 \sigma$ excess above the ambient background and typical Crab signals. Such detections of flares at the level of $20-30$ Crab units could prompt follow-up observations from other high-energy observatories of the flaring system, making CALET a valuable monitoring system.

\section{Summary}

The CALET calorimeter will perform very well in the identification of a gamma-ray dataset, including rejection of contaminating electrons at better than 1 in $10^{3}$. This rejection factor is expected to improve further with the inclusion of IMC data in the tracking and separation algorithms. CALET will be able to observe bright, steady gamma-ray sources, with sensitivity up to TeV energies. Furthermore, CALET will be a very useful instrument for all-sky detection and observation of flares at $\geq 100 \mathrm{GeV}$ energies from transient systems due to its large field of view and high orbital inclination aboard the ISS. 


\section{Acknowledgements}

The CALET experiment is supported by NASA in the United States, JAXA in Japan, and ASI in Italy. Portions of this research were conducted with high performance computing resources provided by Louisiana State University (http://www.hpc.lsu.edu). AGI Satellite Toolkit (STK) software was used in the generation of some results in this paper. The authors also appreciate the efforts of the Fermi-LAT team in development of the background models used. Reference was made to the University of Chicago TeVCat catalog for collections of source-related information. Financial support for the lead author partially provided by a Louisiana State University Board of Regents Graduate Fellowship.

\section{References}

[1] Shoji Torii for the CALET Collaboration, The Calorimetric Electron Telescope (CALET) for High Energy Astroparticle Physics on the International Space Station, Proc. 33rd ICRC (Rio de Janeiro), 2013, and these proceedings.

[2] K. Kasahara, Home Page for EPICS and Cosmos, [http: / / cosmos.n. kanagawa-u.ac.jp/]

[3] Masaki Mori for the CALET Collaboration, Expected Performance of CALET as a High Energy Gamma Ray Observatory, Proc. 33rd ICRC (Rio de Janeiro), 2013.

[4] Fermi Science Support Center, Galactic Interstellar Emission Model and Isotropic Spectral Template, LAT Background Models,

[http://fermi.gsfc.nasa.gov/ssc/data/access/lat/BackgroundModels.html]

[5] M. Ackermann et al., The First Fermi-LAT Catalog of Sources above 10 GeV, ApJS, 209, 34 [astro-ph. IM/1306.6772v3], 2013.

[6] P. L. Nolan et al., FERMI LARGE AREA TELESCOPE SECOND SOURCE CATALOG, ApJS, 199 , 31, 2012.

[7] Alex Moiseev for the CALET Collaboration, CALET Perspectives in High-Energy Gamma-Ray Observations, Proc. 33rd ICRC (Rio de Janeiro), 2013.

[8] O. Adriani et al., The cosmic-ray electron flux measured by the PAMELA experiment between 1 and 625 GeV, Phys. Rev. Lett., 106, 20 [astro-ph. HE/1103/2880v1], 2011.

[9] R. Buehler et al., Gamma-ray activity in the Crab Nebula: the exceptional flare of 2011 April, Ap. J., 749, 262012

[10] M. Mayer et al., Rapid gamma-ray flux variability during the 2013 March Crab Nebula flare, Ap. J. L., 775, L37 2013 